\title{
Papers
}

\section{Childhood cancer in relation to distance from high voltage power lines in England and Wales: a case-control study}

\author{
Gerald Draper, Tim Vincent, Mary E Kroll, John Swanson
}

\begin{abstract}
Objective To determine whether there is an association between distance of home address at birth from high voltage power lines and the incidence of leukaemia and other cancers in children in England and Wales.

Design Case-control study.

Setting Cancer registry and National Grid records. Subjects Records of 29081 children with cancer, including 9700 with leukaemia. Children were aged 0-14 years and born in England and Wales, 1962-95. Controls were individually matched for sex, approximate date of birth, and birth registration district. No active participation was required. Main outcome measures Distance from home address at birth to the nearest high voltage overhead power line in existence at the time.

Results Compared with those who lived $>600 \mathrm{~m}$ from a line at birth, children who lived within $200 \mathrm{~m}$ had a relative risk of leukaemia of 1.69 (95\% confidence interval 1.13 to 2.53$)$; those born between 200 and $600 \mathrm{~m}$ had a relative risk of 1.23 (1.02 to 1.49). There was a significant $(\mathrm{P}<0.01)$ trend in risk in relation to the reciprocal of distance from the line. No excess risk in relation to proximity to lines was found for other childhood cancers.

Conclusions There is an association between childhood leukaemia and proximity of home address at birth to high voltage power lines, and the apparent risk extends to a greater distance than would have been expected from previous studies. About 4\% of children in England and Wales live within $600 \mathrm{~m}$ of high voltage lines at birth. If the association is causal, about 1\% of childhood leukaemia in England and Wales would be attributable to these lines, though this estimate has considerable statistical uncertainty. There is no accepted biological mechanism to explain the epidemiological results; indeed, the relation may be due to chance or confounding.
\end{abstract}

\section{Introduction}

The electric power system produces extremely low frequency electric and magnetic fields. Since 1979 there has been concern that these fields may be associated with cancer. ${ }^{1}$ Concern has concentrated on magnetic rather than electric fields and on childhood leukaemia in particular. A pooled analysis of nine studies that met specified quality criteria found that children living in homes with 24 hour average fields of $\geq 0.4 \mu \mathrm{T}$ have twice the risk of leukaemia. ${ }^{2}$ In 2001 the International Agency for Research on Cancer classified extremely low frequency magnetic fields as "possibly carcinogenic" on the basis of "limited" epidemiological evidence and "inadequate" evidence from animals.

Magnetic fields in homes arise mainly from low voltage distribution wiring, house wiring, and domestic appliances. Only a small fraction of homes are close to high voltage overhead power lines (transmission lines), but in these homes the power line is likely to be the main source of magnetic field.

We investigated whether proximity of home address at birth to transmission lines in England and Wales is associated with increased risks of childhood cancer. It is not known which period of life, if any, is relevant to induction of cancer by magnetic fields. Previous research has considered address at diagnosis or throughout some specified period. Over half $(55 \%)$ of cases of childhood leukaemia and $43 \%$ of other cancers in childhood occur by the age of 5 years.

\section{Methods}

\section{Cases and controls}

Children aged 0-14 years with cancer (malignant neoplasms and tumours of the central nervous system and brain) in England, Scotland, and Wales, ascertained through several sources including the National Cancer Registration System and the UK Children's Cancer Study Group, are included in the National Registry of Childhood Tumours at the Childhood Cancer Research Group.

We identified nearly 33000 cases of childhood cancer in children born in England and Wales, 1962-95, and diagnosed in England, Wales, or Scotland over the same period. We obtained birth information for just over 31000 cases, 1700 having been excluded because the child was adopted or the birth record could not be traced. For each case we selected from birth registers a control matched for sex, date of birth (within six months), and birth registration district. Registration districts vary greatly in size and are frequently redefined; there are currently about 400. We attempted to find the postcode and approximate grid reference of the address at birth for all cases and controls, but this was not always possible. The final dataset comprised 29081 matched case-control pairs (9700 for leukaemia) that we could map with respect to transmission lines.

\section{Calculation of distance from power lines}

We looked at overhead power lines forming the National Grid in England and Wales-that is, all 275 and $400 \mathrm{kV}$ overhead lines (the highest voltages used) plus a small fraction of $132 \mathrm{kV}$ lines, about $7000 \mathrm{~km}$ altogether. We obtained the grid references of all 21800 pylons concerned from the records of National Grid Transco. Using the postcode at birth we identified subjects living within $1 \mathrm{~km}$ of a transmission line. For $93 \%$ of these addresses we 


\begin{tabular}{|c|c|c|c|c|c|c|c|c|c|}
\hline \multirow[b]{2}{*}{ Distance to line (metres) } & \multicolumn{3}{|c|}{ Leukaemia } & \multicolumn{3}{|c|}{ CNS/brain tumours } & \multicolumn{3}{|c|}{ Other diagnoses } \\
\hline & Cases & Controls & RR & Cases & Controls & RR & Cases & Controls & RR \\
\hline $0-49$ & 5 & 3 & 1.67 & 3 & 7 & 0.44 & 7 & 6 & 1.17 \\
\hline $50-99$ & 19 & 11 & 1.79 & 4 & 6 & 0.69 & 15 & 16 & 0.91 \\
\hline 100-199 & 40 & 25 & 1.64 & 26 & 32 & 0.82 & 37 & 45 & 0.81 \\
\hline $200-299$ & 44 & 39 & 1.16 & 38 & 28 & 1.35 & 66 & 76 & 0.87 \\
\hline $300-399$ & 61 & 54 & 1.15 & 35 & 30 & 1.19 & 79 & 65 & 1.21 \\
\hline $400-499$ & 78 & 65 & 1.23 & 40 & 42 & 0.96 & 80 & 97 & 0.82 \\
\hline $500-599$ & 75 & 56 & 1.36 & 54 & 41 & 1.33 & 86 & 85 & 1.01 \\
\hline$\geq 600$ (reference group) & 9378 & 9447 & 1.00 & 6405 & 6419 & 1.00 & 12406 & 12386 & 1.00 \\
\hline Total & 9700 & 9700 & & 6605 & 6605 & & 12776 & 12776 & \\
\hline
\end{tabular}

CNS=central nervous system.

obtained, from the Ordnance Survey product AddressPoint, a $0.1 \mathrm{~m}$ grid reference and hence calculated the shortest distance to any of the transmission lines that had existed in the year of birth, re-creating previous locations of lines when necessary and possible. For calculated distances less than $50 \mathrm{~m}$, we took the average of the nearest and furthest points of the building from the line, using large scale maps. We aimed to obtain a complete set of accurate distances for all subjects within $600 \mathrm{~m}$ of a line, a distance chosen to be well beyond that at which the magnetic field from the line is thought to be important.

\section{Statistical analysis}

We used conditional logistic regression on the matched case-control pairs to calculate relative risks and $\chi^{2}$ values.

\section{Results}

Table 1 shows the distribution of distances from the nearest line for cases, subdivided into leukaemia, central nervous system/ brain, and "other," and for matched controls. Most (97\%) of these distances were $\geq 600 \mathrm{~m}$. The relative risk is an estimate of the incidence compared with that at distances $\geq 600 \mathrm{~m}$. For leukaemia, at each distance category $<600 \mathrm{~m}$ the relative risks are greater than 1.0; there is some evidence that the risk varies according to distance from the line, though there is no smooth trend. For the other diagnoses, our data suggest no increased risk.

In general, emanations from a line source are expected to reduce in strength as the reciprocal of distance, but the magnetic field from a power line generally falls as the inverse square of distance, or sometimes the inverse cube. ${ }^{3}$ For each diagnostic group, we tested whether the risk is some function of distance $(d)$ from the nearest line (table 2), using three models: that the risk depends on the rank of the distance band, the reciprocal of the distance $(1 / d)$, or the inverse square $\left(1 / d^{2}\right)$. There were no significant results for central nervous system/brain tumours or for "other tumours." For leukaemia, the results of two of the trend analyses were significant $(\mathrm{P}<0.01)$; these analyses suggest the risk might depend either on the rank of the distance category or on the reciprocal of distance. The latter seems more plausible. We therefore retabulated the results for leukaemia at intervals corresponding to roughly equal intervals of $1 / d$ (table 3 ). This change in the grouping of the data does not change the pattern of relative risk estimates shown in table 1 or the significance of the test for trend with $1 / d$. For simplicity we also analysed risk of leukaemia in bands 0-199 $\mathrm{m}$ and $200-599 \mathrm{~m}$. The risks relative to $\geq 600 \mathrm{~m}$ were 1.69 and 1.23; the trend with $1 / d$ was significant $(\mathrm{P}<0.01)$.

We examined the possibility that the relation between distance and risk of leukaemia is a consequence of a relation between distance and socioeconomic status. We used the Carstairs deprivation index to allocate a measure of socioeconomic status to the census ward in which each child was living at birth. ${ }^{4}$ The results in table 4 confirm the previously reported association between affluence and risk of childhood leukaemia (P for trend $<0.01) .{ }^{5}$ Adjustment for socioeconomic status had no effect on the relative risks for distance (table 3).

Power lines produce small air ions through a process known as "corona." Fews et al suggest that this could lead to health

Table 2 Tests of hypotheses relating trends in relative risks to alternative measures of proximity to nearest line (based on the eight distance categories ${ }^{*}$ in table 1). Figures are $\chi^{2}$ for trend (with $1 \mathrm{df}$ ) and $P$ value

\begin{tabular}{|c|c|c|c|}
\hline & Leukaemia & CNS/brain tumours & Other diagnoses \\
\hline Ranked distances & $8.76, P=0.003$ & $0.01, P=0.924$ & $0.64, P=0.424$ \\
\hline Reciprocal of distance $(1 / d)$ & $6.72, P=0.0095$ & 1.09, $P=0.296$ & $0.12, P=0.733$ \\
\hline Reciprocal of square of distance $\left(1 / d^{2}\right)$ & $1.47, P=0.225$ & $1.83, P=0.177$ & $0.03, P=0.873$ \\
\hline
\end{tabular}

*Distance $(d)$ for each case is taken as midpoint of limits of band within which it lies (as specified in table 1).

Table 3 Relative risk (RR) estimates for leukaemia using revised distance categories (see text)

\begin{tabular}{|c|c|c|c|}
\hline Distance, $d$ (metres) & $1 / d$ & RR (95\% CI) & $\mathrm{RR}^{*}(95 \% \mathrm{Cl})$ \\
\hline $0-49$ & 0.040 & 1.67 (0.40 to 6.97) & 1.65 (0.39 to 6.89$)$ \\
\hline $50-69$ & 0.017 & 1.51 (0.48 to 4.79$)$ & 1.53 (0.48 to 4.83$)$ \\
\hline $70-99$ & 0.012 & 2.02 (0.76 to 5.39$)$ & 2.00 (0.75 to 5.32) \\
\hline 100-199 & 0.007 & 1.64 (1.00 to 2.71$)$ & 1.64 (0.99 to 2.70) \\
\hline$\overline{0-199}$ & 0.010 & 1.69 (1.13 to 2.53$)$ & 1.68 (1.12 to 2.52$)$ \\
\hline $200-599$ & 0.003 & 1.23 (1.02 to 1.49$)$ & 1.22 (1.01 to 1.47$)$ \\
\hline$\geq 600$ (reference group) & 0.000 & 1.00 & 1.00 \\
\hline
\end{tabular}

*Adjusted for socioeconomic status. 
Table 4 Relative risks for categories of socioeconomic status

\begin{tabular}{lccc} 
Socioeconomic status & Leukaemia & CNS/brain tumours & Other diagnoses \\
\hline 1 (most affluent) & 1.00 & 1.00 & 1.00 \\
\hline 2 & 0.96 & 0.97 & 1.04 \\
\hline 3 & 0.94 & 0.93 & 0.99 \\
\hline 4 & 0.90 & 0.97 & 0.95 \\
\hline 5 (most deprived) & 0.88 & 0.92 & 0.98 \\
\hline$\chi^{2}$ for trend & $6.79, P=0.009$ & $1.38, P=0.240$ & $1.07, P=0.302$ \\
\hline
\end{tabular}

effects when winds blow the ions away from the line. ${ }^{6}$ We have made an initial test of this hypothesis using a simple model suggested by Preece et al (personal communication), assuming the prevailing wind is from the south west. The case-control ratio was no greater downwind than upwind of power lines, so, using this admittedly oversimplified approach, we have no evidence to support this hypothesis.

\section{Discussion}

To date this is the largest study of childhood cancer and power lines, with roughly twice the number of children living close to power lines than in the next largest study. ${ }^{7}$ We found that the relative risk of leukaemia was 1.69 (95\% confidence interval 1.13 to 2.53) for children whose home address at birth was within 200 $\mathrm{m}$ of a high voltage power line compared with those more than $600 \mathrm{~m}$ from the nearest line. For $200-600 \mathrm{~m}$ the relative risk was 1.23 (1.02 to 1.49). The finding that the increased leukaemia risk apparently extends so far from the line is surprising in view of the very low level of magnetic field that could be produced by power lines at these distances.

\section{Possible explanations for findings}

There is no obvious source of bias in the choice of cases or controls. The study is based on records of childhood cancer in England and Wales over most of the period that the National Grid has existed. Registration for childhood cancer is nearly complete, and it seems improbable that the likelihood of registration is related to proximity of birth address to transmission lines. Controls were selected from registers compiled through the legally required process of birth registration. No participation by cases or controls was required. We calculated distances without knowing case-control status, and we were able to include $88 \%$ of the eligible cases, each with a matched control.

Populations near power lines may have different characteristics from the rest of the population. In our control data there is a slight tendency in urban areas for greater affluence (measured by the Carstairs index) closer to lines, though in rural areas there is no clear trend. There is known to be a positive association between affluence and rates of childhood leukaemia. However, adjustment for socioeconomic status of the census ward of birth address did not explain our finding. Population mixing has been associated with childhood leukaemia, ${ }^{8}$ but in our cases individual mobility, measured by changes of postcode between birth and diagnosis, was no more common for those whose home at birth was closer to the lines. Other characteristics of the population (for instance parity, which has sometimes been found to be associated with childhood leukaemia ${ }^{9}$ ) may vary with proximity to power lines, but we do not have the data to determine whether these explain our result.

The results are highly significant but could nevertheless be due to chance-for example, if the leukaemia controls are not sufficiently representative of the relevant population. Some support for this explanation can be derived from the different distance distributions observed for the leukaemia and nonleukaemia controls in table 1. Comparison of the leukaemia cases with the latter still suggests that there is an increased risk for leukaemia but it is much lower than that found using the matched controls. We emphasise, however, that the use of the matched controls is the most appropriate approach.

Six of the studies included in the pooled analysis referred to above $^{2}$ contain, or have been extended to include, analyses of proximity to power lines. ${ }^{70-14}$ Of these, one, a previous UK study, ${ }^{10}$ with 1582 cases of leukaemia diagnosed during 1992-6 (most of which will be contained within our 9700), found a relative risk of 1.42 (0.85 to 2.37) for acute lymphocytic leukaemia within $400 \mathrm{~m}$ for 275 and $400 \mathrm{kV}$ lines; this supports our results. Studies in Canada ${ }^{11}$ and Sweden ${ }^{7}$ also found increased risks for childhood leukaemia (Canada: relative risk 1.8 (0.7 to 4.7) for residence within $100 \mathrm{~m}$ of transmission lines of $50 \mathrm{kV}$ or more, and 1.3 within $50 \mathrm{~m}$; Sweden: 2.9 (1.0 to 7.3) for residence $\leq 50$ $\mathrm{m}$ versus $101-300 \mathrm{~m}$ from 220 and $400 \mathrm{kV}$ power lines, with no increase for other childhood cancers). Studies from Denmark, ${ }^{12}$ Norway, ${ }^{13}$ and the United States ${ }^{14}$ found relative risks below 1.0 but were based on smaller numbers. None of these estimates relates to distances as great as ours; some used a reference category that is within the distance where we found an increased risk.

Our study concerned home address at birth, whereas much previous magnetic field epidemiology has concerned address at other times. Half of the children with leukaemia in this study had the same address at diagnosis as at birth; we have no corresponding information for the control group.

The most obvious explanation of the association with distance from a line is that it is indeed a consequence of exposure to magnetic fields. For magnetic fields in the home the pooled analysis by Ahlbom et al found a relative risk of 2.00 (1.27 to 3.13) for exposures $\geq 0.4 \mu \mathrm{T}$ versus $<0.1 \mu \mathrm{T}$; the risks for fields $<0.4 \mu \mathrm{T}$ were near the no effect level. ${ }^{2}$ Another pooled analysis, including additional studies, found a similar result with a threshold of $0.3 \mu \mathrm{T} .^{15}$ For the power lines we investigated, the magnetic field falls to $0.4 \mu \mathrm{T}$ at an average of about $60 \mathrm{~m}$ from the line (based on calculations using one year of recorded loads for a sample of 42 lines). Our increased risk seems to extend to at least $200 \mathrm{~m}$, and at that distance typical calculated fields from power lines are $<0.1 \mu \mathrm{T}$, and often $<0.01 \mu \mathrm{T}$-that is, less than the average fields in homes from other sources. Thus our results do not seem to be compatible with the existing data on the relation between magnetic fields and risk. The estimated relative risk was more closely related to the reciprocal of the distance from the line than to the square of the reciprocal of the distance.

\section{Conclusions}

While few children in England and Wales live close to high voltage power lines at birth, there is a slight tendency for the birth addresses of children with leukaemia to be closer to these lines than those of matched controls. An association between childhood leukaemia and power lines has been reported in several studies, but it is nevertheless surprising to find the effect extending so far from the lines. We have no satisfactory explanation for our results in terms of causation by magnetic fields or association with other factors. Neither the association reported here nor previous findings relating to level of exposure to magnetic fields are supported by convincing laboratory data or any accepted biological mechanism.

Assuming that the higher risk in the vicinity of high voltage lines is indeed a consequence of proximity to the lines we can estimate the attributable annual number of cases of childhood 


\section{What is already known on this topic}

Power frequency magnetic fields, produced by the electric power system, are "possibly carcinogenic"

A pooled analysis of case-control studies found that children living in homes with high magnetic fields $(>0.4$ $\mu \mathrm{T})$ had twice the risk of childhood leukaemia

High voltage power lines are one source of these fields What this study adds

A UK study of 29000 cases of childhood cancer, including 9700 cases of leukaemia, found a raised risk of childhood leukaemia in children who lived within $200 \mathrm{~m}$ of high voltage lines at birth compared with those who lived beyond $600 \mathrm{~m}$ (relative risk 1.7 )

There was also a slightly increased risk for those living 200-600 $\mathrm{m}$ from the lines at birth (relative risk 1.2, P for trend $<0.01$ ); as this is further than can readily be explained by magnetic fields it may be due to other aetiological factors associated with power lines

leukaemia in England and Wales. The annual incidence of childhood leukaemia in England and Wales is about 42 per million; the excess relative risks at distances of $0-199 \mathrm{~m}$ and $200-599 \mathrm{~m}$ are about 0.69 and 0.23 , respectively, giving excess rates of 28 and 10 per million. (These two estimates allow for the fact that the incidence for England and Wales is itself partly based on cases occurring in the vicinity of power lines.) We estimate that of the 9.7 million children in the population (2003 estimate), at birth about 80000 would have lived within $199 \mathrm{~m}$ of a line and 320000 between 200 and $599 \mathrm{~m}$. Thus, of the 400420 cases of childhood leukaemia occurring annually, about five would be associated with high voltage power lines, though this estimate is imprecise. We emphasise again the uncertainty about whether this statistical association represents a causal relation.

We are grateful to colleagues at the Childhood Cancer Research Group and at National Grid Transco for help with this study and to cancer registries and the United Kingdom Children's Cancer Study Group for notifications of cases of childhood cancer.

Contributors: GD was responsible for overall direction of the study and publication. GD and JS had the initial idea and designed the study. TV and MEK collected information on cases and controls and carried out the statistical analysis. JS assessed exposures. GD and JS are guarantors

Funding: This study was undertaken as part of a project funded by the United Kingdom Department of Health Radiation Protection Programme. The Childhood Cancer Research Group also receives funding from the

\section{Science commentary: Power to confuse}

Geoff Watts

Ever since Nancy Wertheimer of the University of Colorado reported her 1979 findings of an excess of cancer in children living near overhead power lines, seldom has a year passed without a flurry of public debate over the safety or otherwise of these ugly (the one thing all parties agree on) but essential installations.

Much of the argument has been about the very existence of the alleged hazard. As recently as last month, the organisers of
Department of Health and the Scottish Ministers. The views expressed here are those of the authors and not necessarily those of the Department of Health and the Scottish Ministers. National Grid Transco provided staff time but no other funding.

Competing interests: JS is employed by National Grid Transco and worked on this project with their permission. A written contract exists between the Childhood Cancer Research Group and National Grid Transco specifying that the Childhood Cancer Research Group has complete control over the conduct, interpretation, and publication of this study; this paper has not been approved by anyone in National Grid Transco other than JS in his capacity as author and does not necessarily represent National Grid Transco's views.

Ethical approval: The Childhood Cancer Research Group has local ethics committee approval and, through membership of the UK Association of Cancer Registries, has approval from the Patient Information Advisory Group with respect to cancer registration function.

1 Wertheimer N, Leeper E. Electrical wiring configurations and childhood cancer. Am J Epidemiol 1979;109:273-84.

Ahlbom A, Day N, Feychting M, Roman E, Skinner J, Dockerty J, et al. A pooled analysis of magnetic fields and childhood leukaemia. BrJ Cancer 2000;83:692-8.

3 Maddock BJ. Overhead line design in relation to electric and magnetic field limits. Power Engineering 1992;Sep:217-24.

4 Morris R, Carstairs V. Which deprivation? A comparison of selected deprivation indexes. J Public Health Med 1991;13:318-26.

5 Draper GJ, Stiller CA, O'Connor CM, Vincent TJ, Elliott P, McGale P, et al. The geographical epidemiology of childhood leukaemia and non-Hodgkin lymphomas in Great Britain, 1966-83. London: Office for Population Census and Surveys, 1991. (OPCS Studies on Medical and Population Subjects No 53.)

6 Fews AP, Henshaw DL, Wilding RJ, Keitch PA. Corona ions from powerlines and increased exposure to pollutant aerosols. Int J Radiat Biol 1999;75:1523-31.

7 Feychting M, Ahlbom A. Magnetic fields and cancer in children residing near Swedish high-voltage power lines. Am J Epidemiol 1993;138:467-81.

8 Kinlen L, Doll R. Population mixing and childhood leukaemia: Fallon and other US clusters. BrJ Cancer 2004;91:1-3.

9 Dockerty JD, Draper GJ, Vincent TJ, Rowan SD, Bunch KJ. Case-control study of parental age, parity and socioeconomic level in relation to childhood cancers. Int J Epidemiol 2001;30:1428-37.

10 UK Childhood Cancer Study Investigators. Childhood cancer and residential proximity to power lines. Br J Cancer 2000;83:1573-80.

11 McBride ML, Gallagher RP, Thériault G, Armstrong BG, Tamaro S, Spinelli JJ, et al. Power-frequency electric and magnetic fields and risk of childhood leukemia in Power-frequency electric and magnetic
Canada. Am J Epidemiol 1999;149:831-42.

12 Olsen JH, Nielsen A, Schulgen G. Residence near high voltage facilities and risk of cancer in children. BMJ 1993;307:891-5.

13 Tynes T, Haldorsen T. Electromagnetic fields and cancer in children residing near Norwegian high-voltage power lines. Am J Epidemiol 1997;145:219-26.

14 Kleinerman RA, Kaune WT, Hatch EE, Wacholder S, Linet MS, Robison LL, et al. Are children living near high-voltage power lines at increased risk of acute lymphoblastic leukemia? Am J Epidemiol 2000;151:512-5.

15 Greenland S, Sheppard AR, Kaune WT, Poole C, Kelsh MA. A pooled analysis of magnetic fields, wire codes, and childhood leukemia. Epidemiology 2000;11:624-34. (Accepted 6 April 2005)

bmj.com 2005;330:1290

Childhood Cancer Research Group, University of Oxford, Oxford OX2 6HJ Gerald Draper homorary senior research fellow

Tim Vincent research officer

Mary E Kroll statistician

National Grid Transco plc, London WC2N 5EH

John Swanson scientific adviser

Correspondence to: GJ Draper gerald.draper@

ccrg.ox.ac.uk

the 15 year UK childhood cancer study declared that "perceived risk factors such as living near sources of electromagnetic fields . .. are not principal causes, if at all, of leukaemia in children." But a clutch of studies reporting a positive association-of which this week's by Draper and colleagues is the most recent ${ }^{1}$-has encouraged researchers to continue investigating possible mechanisms. 
Electrical and magnetic fields can induce currents that might alter the voltages across cell membranes. Magnetic fields might cause the movement of ferromagnetic particles within cells. They might also influence free radicals: atoms with unpaired electrons that are highly reactive and play a part in all sorts of biochemical processes. Low frequency electromagnetic fields have been said to alter the progress of cells through the cell cycle and reduce the effectiveness of the immune system. Power lines might even deflect and concentrate cosmic rays on people living within their vicinity. Evidence to support these and other ideas, however, is at best thin and at worst non-existent.

One of the more recent attempts at identifying a mechanism sidesteps the need to invoke direct effects. For the past 10 years or so, Bristol University physicist Dennis Henshaw has been working on the influence of powerful electric fields on the deposition of airborne particles. The relevance of this to power lines entered public consciousness in 1999 with the publication of two papers by Henshaw and colleagues. ${ }^{2}{ }^{3}$ High energy power systems, they pointed out, cause some breakdown in the surrounding air molecules and so generate positive or negative ions. The systems are designed to minimise this effect, but it does still occur-and any aerosol pollutants that pass through these ion clouds can acquire an electrical charge.

If particles with a charge are inhaled, more of them will stick to the lining of the respiratory system. The data are limited, but one study that used a model of the human airway suggests that deposition could be increased by a factor of around three. A 2004 report by the (then) National Radiological Protection Board conceded the plausibility of the mechanism and suggested some further experiments. ${ }^{4}$ Draper and colleagues refer to the Henshaw hypothesis but add that more work will be necessary to rule it in or out. ${ }^{1}$

Like the fluoridation of drinking water and the genetic modification of crops, the debate over power lines seems destined to be with us for a while yet. So, in these risk averse times, and before activists begin blowing up pylons, a bit of perspective might help. In 2002, according to the Child Accident Prevention Trust, more than 36000 children were hurt in road accidents and around 200 were killed. Another 32 died in house fires. Draper and colleagues reckon that five cases annually of childhood leukaemia may be associated with power lines.

1 Draper G, Vincent T, Kroll ME, Swanson J. Childhood cancer in relation to distance from high voltage power lines in England and Wales: a case-control study. BMJ 2005;330:page nos.

2 Fews AP, Henshaw DL, Keitch PA, Close JJ, Wilding RJ. Increased exposure to pollutant aerosols under high voltage powerlines. Int J Radiat Biol 1999;75:1505-21.

3 Fews AP, Henshaw DL, Wilding RJ, Keitch PA. Corona ions from powerlines and increased exposure to pollutant aerosols. Int J Radiat Biol 1999;75:1523-31.

4 National Radiological Protection Board. Particle deposition in the vicinity of power lines and possible effects on health. Documents of NRPB 2004;15(1).

28 New End Square, London NW3 1LS

Geoff Watts science editor, BMI

geoff@ scileg.freeserve.co.uk 